\title{
La situación de la teoría y terminología de la psicología popular y el futuro de la psicología científica*
}

Ricardo Braun

En este artículo se analiza el origen y la función de la denominada psicología popular -folk psychology-y su posible inclusión en la psicología cientifica. Se aborda el problema del reduccionismo en la ciencia y del eliminativismo, defendiendo una postura materialista no-reduccionista de la psicología que reivindica el estatus de la psicología popular y su rol en la psicología. En el artículo se exploran y cuestionan la tesis de la unidad de la ciencia y el reduccionismo, analizando el problema de la microrreducción en la fisica, para concluir que el reduccionismo en la ciencia limita y sobresimplifica la compleja realidad. En el caso de la psicología popular, como fuente de explicaciones no reducibles, tiene un rol en el desarrollo de la psicología científica que se puede entender a partir de una propuesta de dos usos posibles.

psicología popular /reduccionismo / eliminativismo / psicología científica

\section{The status of the theory and terminology of folk psychology and the future of scientific psychology}

This article discusses the origins and function of folk psychology and its assimilation in a future scientific psychology. The problems of reductionism in science and eliminativism in psychology are analyzed. The author defends a non-reductive materialism view of psychology and argues for a vindication of the status and role of folk psychology. In this article the unity of science thesis and reductionism are explored and questioned to conclude that taking as an example, the problem of micro-reductions in physics. It is proposed, in contrast, a pluralist standpoint whereby the autonomy and interrelation of the different levels of reality are maintained. The article defends the role of folk psychology, as a source of non-reductive explanations, in the growth of scientific psychology which can be understood in a proposal of two possible uses.

folk psychology / reductionism / eliminativism /scientific psychology

* Este artículo es resultado del avance de la investigación "Psicología popular y psicología científica", auspiciada por el Instituto de Investigación Científica de la Universidad de Lima. Agradezco al Instituto por el apoyo para la realización de este estudio y por la autorización para publicar parte del trabajo.

Correo electrónico: rbraun@correo.ulima.edu.pe 
Mucho se ha escrito en los últimos veinte años acerca del estatus, naturaleza y perspectivas futuras de la llamada psicología popular o folk psychology en el vocabulario anglosajón. Mi propósito es introducir a la comunidad psicológica un área de estudio de la filosofía de la mente que es muy activa actualmente por las implicancias que conlleva en la epistemología de la psicología científica. Para ello, resumiré los antecedentes y analizaré brevemente el problema de la psicología popular, para luego discutir las perspectivas del lugar de la psicología popular en la psicología científica futura. En lo que sigue, defenderé la posición a favor del mantenimiento de la psicología popular dentro de la psicología científica. Parto de la concepción denominada materialismo no-reduccionista y argumentaré por el no-reduccionismo como estrategia en las ciencias en general y en la psicología en particular, para luego presentar argumentos específicos en defensa de la psicología popular.

\section{ORIGEN DE LA PSICOLOGÍA POPULAR}

¿Qué es exactamente la psicología popular? La psicología popular es un conjunto de conceptos interrelacionados que usan las personas ordinarias para comprender, explicar y predecir el comportamiento y estados mentales propios y ajenos. En todas las lenguas hay una serie de términos que forman parte de nuestro vocabulario ordinario, es decir, no técnico, a través de los cua- les describimos nuestra llamada "vida mental". Algunos de ellos son "deseo", "creencia", "intención", "sensación", "miedo", "amor", "temor".

Los seres humanos somos seres sociales y criaturas reflexivas. Como tales, continuamente participamos en una serie de prácticas cognitivas que nos permite participar del mundo social. En particular, tratan de entender, explicar y predecir sus estados psicológicos y comportamiento manifiesto y el de otros. Para hacerlo emplean una serie de conceptos como los mencionados, que se refieren a los estados mentales tanto actuales como disposicionales. La comprensión social y la coordinación social dependen de los deseos y las creencias: las utilizamos para interactuar con los demás. Es parte de la discusión acerca de la psicología popular si ella constituye una teoría, en el sentido que constituye un marco conceptual o red de principios que se usa para entender, explicar y predecir las conductas propias y ajenas. Precisamente, parte del problema es determinar si constituye una teoría o prototeoría psicológica o no (von Eckardt, 1994).

El término "psicología popular" como una teoría psicológica popular tiene origen en los trabajos del filósofo Wilfred Sellars y en su argumento en contra del "mito de lo dado" (1968). Como un ataque a las teorías acerca del conocimiento inmediato de los datos sensoriales propuesto por el empirismo 
lógico, Sellars sostenía que ninguna prueba sensorial aparece de la manera que garantizaría la verdad de las proposiciones que la describen. En otras palabras, el mito consistía en que se podía verificar empíricamente la presencia del objeto de una experiencia sensible, independiente de la palabra explícita o pensada implícitamente, puesto que es dicho objeto el que me permite tener la experiencia. Aplicado a la teoría de la mente, Sellars negaba que el contenido de nuestra vida mental se nos presentara inequívocamente. De esta manera también negaba el acceso epistémico privilegiado que tienen nuestros estados mentales. Para contrarrestar el "mito de lo dado" Sellars inventa otro mito en el que nuestros ancestros, limitados a una comprensión puramente conductista de las acciones, crearon una teoría de la acción que postulaba estados internos como causas del comportamiento observado. Al principio nuestros ancestros utilizaron este nuevo mito para aplicarlo a otros, pero pronto aprendieron a comprender sus propios estados mentales a partir de su comportamiento: nacían también los estados mentales autoadscritos. Con el transcurso del tiempo, los ancestros se convirtieron en expertos en atribuir estados mentales internos a su propia conducta y a la ajena. Si bien no desarrollaron una teoría, los estados internos que se atribuían a sí mismos se convirtieron en los entes que se postularían en una teoría de la mente. Es obvio que Sellars no pretendía hacer una historiografía del origen de nuestra atribución de estados internos, sino simplemente retomar el debate acerca de la atribución y uso de los estados mentales internos.

Durante décadas, la psicología científica no pareció muy interesada en el estudio de la psicología popular, puesto que bajo el dominio de cualquier forma de conductismo, los psicólogos no tuvieron como campo de estudio estados internos, que son característicos de la psicología popular. En efecto, los estados internos fueron considerados básicamente como "ficciones explicativas" de acuerdo a la posición skinneriana. La consecuencia lógica fue la exclusión metodológica de teorías cognitivas sustantivas que hicieran referencia a estados psicológicos con contenido y eficaces causalmente. Las únicas discusiones filosóficas relevantes para la psicología científica eran las justificaciones filosóficas del conductismo lógico como el de Ryle o las críticas a los principios de la teoría del condicionamiento conductual.

Sin embargo, con el advenimiento de la psicología cognitiva, nuevamente abrió la necesidad del análisis de las teorías cognitiva basadas en la perspectiva del procesamiento de información. Hubo entonces un poderoso ímpetu para el desarrollo del estudio de la inteligencia artificial en computadoras, pro- 
porcionando a los psicólogos de un modelo teórico de la cognición humana en términos de programas de procesamiento interno. El éxito de las teorías cognitivas basadas en este enfoque de procesamiento de información y la habilidad para simular tareas cognitivas en computadoras, convencieron a muchos de la utilidad de los constructos teóricos de la psicología y la realidad de las estructuras y procesos que se les asignaban. Como dijo Neisser (1967): "la razón básica para el estudio de los procesos cognitivos se volvió tan clara como la razón para estudiar cualquier cosa: porque estaban alli".

La psicología cognitiva fue quizás el movimiento que promovió más la concepción de que nuestra comprensión cotidiana de nuestros estados mentales constituye una teoría popular acerca de la mente. En efecto, el desarrollo del cognitivismo provocó la postulación de los estados internos como causantes del comportamiento interno y externo. Los estados internos fueron tomados como representaciones (computacionalmente manipulables) y se empezó a utilizar el término "teoría" para referirse a las estructuras representacionales de suficiente complejidad. Este uso del término "teoría" es paralelo al que los cognitivistas aplicaron para explicar el manejo que tenemos los seres humanos de los objetos cotidianos. Los seres humanos tenemos una "teoría dinámica" internamente representada que nos proporciona un marco para entender cómo funcionan los objetos físicos. A esa teoría física la denominarían "física popular" (folk physics) (McCloskey, 1983). De forma análoga, los cognitivistas adoptaron una estrategia similar, suponiendo que las personas utilizan una teoría que postula la existencia de representaciones internas cuando se trata de explicar la capacidad popular para explicar y predecir comportamientos. A esta teoría se le vino a llamar "psicología popular" (folk psychology) (Ravenscroft, 2004).

En la actualidad la ciencia cognitiva, la disciplina que pretende entender los problemas acerca del funcionamiento mental, particularmente el cognoscitivo (adquisición, almacenamiento, y uso de la actividad inteligente) hace uso de categorías que son paralelas a las utilizadas en la psicología popular, las llamadas "actitudes proposicionales", como por ejemplo, cuando decimos que Juan "cree que hoy es lunes". La actitud "cree" tiene como contenido "el que hoy sea lunes". Se dice que las actitudes proposicionales tienen contenido cuando afirmamos que son semánticamente evaluables, y además que son eficaces causalmente, es decir, provocan conductas $u$ otros estados mentales.

Ahora bien, mientras que los conductistas fueron responsables por abandonar las referencias explicativas de los estados psicológicos internos debido a bases epistemológicas cuestionables, la ciencia cognitiva actual podría estar 
equivocada también en emplear las actitudes proposicionales en las explicaciones de la acción humana. Esta es precisamente la crítica de la psicología popular en nuestros días. Se sostiene que el futuro de la psicología popular está en peligro puesto que se dice que nuestras explicaciones basadas en términos de la psicología popular demostrarán ser inferiores o inadecuadas a otras alternativas teóricas basadas en términos neurofisiológicos. Si este resulta ser el caso, entonces nuestras muy familiares explicaciones psicológicas populares deberán ser abandonadas junto con la ontología que las supone. Se argumentó, adicionalmente, que las entidades postuladas por las mejores teorías de la ciencia cognitiva no tendrán identificación alguna con las entidades de nuestras explicaciones populares, como las creencias y deseos. Aún más, los términos que hacen referencia a los fenómenos intencionales no se reducirán paulatinamente a las categorías neurofisiológicas sino que las categorías de la psicología popular se mantendrán aisladas junto con otras como el flogisto, el éter y los demonios, sin ninguna perspectiva de reducción. Esto es lo que se denomina en el argot el materialismo eliminativista.

\section{LA TESIS ELIMINATIVISTA}

El materialismo eliminativista tiene una larga tradición filosófica (se podría rastrear desde el pensamiento de Demócrito, pasando por Hobbes) pero en el siglo XX, y con el desarrollo de la neu- rología, el eliminativismo cobró mayor auge. La tesis básica se sostiene en una convicción metafísica, a saber, que lo único real es lo físico o material, y que por lo tanto, si queremos describir algo en términos no-físicos, como los términos mentales, entonces aquello no sería real en absoluto (Feyerabend, 1963; Rorty, 1970). Para Feyerabend, por ejemplo, hablar de la realidad de las experiencias mentales comporta un error conceptual que se origina por el uso del lenguaje ordinario. Rorty, en cambio, sostiene (o al menos sostenía años atrás) que hablar de lo mental es una forma inadecuada de referirse a lo físico, creyendo equivocadamente que lo mental existe.

El eliminativismo no es sino una parte de la estrategia de un modelo reduccionista de la ciencia: si lo mental es físico, entonces debemos en último término reducir el número de entidades y quedarnos solo con entidades que hagan referencia a lo físico. Ahora bien, se puede ser reduccionista a nivel metafísico y también reduccionista a nivel metodológico. El reduccionismo metafísico sostiene que todos los fenómenos son en último término físicos en su naturaleza. El reduccionismo metodológico afirma que en el contexto de las explicaciones científicas es posible hablar de lo mental en términos de las ciencias físicas. Es interesante presentar esta diferencia porque existen las siguientes posibilidades con respecto al reduccionismo de lo mental: 


\begin{tabular}{lcc}
\hline Teorías acerca de lo mental & \multicolumn{2}{c}{ Aspectos } \\
\hline Dualismo & Metafísico & Metodológico \\
Materialismo eliminativista & No-reduccionista & No-reduccionismo \\
Materialismo no-reduccionista & Reduccionista & Reduccionista \\
\hline
\end{tabular}

La primera teoría no requiere mucho comentario, dado que se sostiene que hay dos sustancias incompatibles, no hay reduccionismo metafísico, y menos podría ser metodológico. El materialismo eliminativista es independiente como teoría del materialismo no-reduccionista. Es fácil advertir las consecuencias del materialismo eliminativista puesto que al considerar que todo es materia, entonces no tendría sentido utilizar el lenguaje conceptual que involucraría metodología y vocabulario que no fuera físico.

El materialismo no-reduccionista, en cambio, puede estar convencido de que todo es materia o una propiedad de la materia, pero encuentra dificultades para explicar la realidad mental en términos físicos o considera que el lenguaje de las teorías físicas sería inadecuado para captar la compleja realidad humana, particularmente la realidad cultural humana (Davidson, 1970; Fodor, 1968; Margolis, 1987).

En la actualidad muchos de los que defienden el estatus de la psicología popular se agruparían dentro del materialismo no-reduccionista.
El materialismo eliminativista tiene sus profetas. Los llamados "profetas del eliminativismo" (Blackburn, 1991) pertenecen a uno de dos tipos. Los hay del tipo "Isaías" ("De la planta del pie a la cabeza, no hay en él cosa sana", Is., I: 6), y los del tipo "Juan el Bautista" (“...pero viene el que es más fuerte que yo", Lc, 3: 15). Los profetas tipo "Isaías" descartan la posibilidad de adecuación, reemplazo conceptual o asimilación moderada de la psicología popular en la psicología científica. Los otros profetas esperan al Mesías y buscan una mejora sustancial. Así, los primeros son radicales y no encuentran nada rescatable ni utilizable de la psicología popular. Los segundos aguardan el cumplimiento de una promesa en la que una ciencia más madura como la neurociencia, será capaz de conducirnos a la tierra prometida de la psicología científica. En otras palabras, los tipo "Juan el Bautista" admiten el uso de la psicología popular pero esperan algo mejor. El tipo Isaías está representado en la literatura por Paul Churchland (1981) y Patricia S. Churchland (1986). 


\section{CONTRA EL REDUCCIONISMO}

\section{METODOLÓGICO}

Una larga tradición en la filosofía de la ciencia sostiene que el conocimiento científico debiera ser integrado para contrabalancear la diversa especialización de las diferentes disciplinas científicas. Esta integración es conocida como el proyecto de la "unidad de la ciencia". La idea básica es que las explicaciones debieran ser integradas, o más precisamente, reducidas a un nivel, el nivel físico. Esto significa que las explicaciones expresadas en un lenguaje no físico debieran ser formuladas en el lenguaje de la física. Hay dos supuestos fundamentales que motivan esta tradición: la creencia de que la integración de las diversas explicaciones en una más unificadora, simplifica la ciencia y expande el poder explicativo de los principios científicos; y, la creencia de que no hay nada de importancia explicativa que no pueda ser formulado en último término en el lenguaje de la física.

Estas dos creencias han estado frecuentemente defendidas. La historia de la ciencia proporciona ejemplos de la reducción de una teoría hacia otra (Popper, 1974). La explicación de la termodinámica por la mecánica estadística y la explicación de la óptica física por el electromagnetismo clásico son dos ejemplos muy conocidos del siglo XIX. El éxito de estos y otros ejemplos propulsaron el programa para reducir las explicaciones de una disciplina a otra. Y para muchos hoy día, este programa juega un rol significativo en la investigación y en el desarrollo de la ciencia.

En lo que sigue quiero rebatir la tesis reduccionista y argumentar a favor de la autonomía de niveles. La objeción al reduccionismo proviene de dos frentes: primero, el reduccionismo no aumenta nuestra comprensión de los fenómenos, y en consecuencia, las explicaciones reduccionistas no son mejores explicaciones; $y$, segundo, al contrario de la posición prevaleciente, la física teorética no es incompatible con la microrreducción. Al argumentar en contra del reduccionismo no considero que se deba renunciar a la idea de la unidad de la ciencia. Al contrario, sostengo que puesto que la unidad de la ciencia no implica el reduccionismo, es posible todavía mantener la unidad de la ciencia sin reduccionismo. Brevemente, explicaré algunos valores de esta visión de la unidad de la ciencia.

En su clásico artículo de 1958, "The Unity of Science as a Working Hypothesis", Oppenheim y Putnam esbozaron el programa de la reducción de los términos y leyes de la ciencia a un nivel fundamental de explicación. El programa determina una sucesión de microrreducciones de un nivel de explicación a otro más básico. Los niveles jerárquicos son de más alto a más bajo: grupos sociales, seres humanos individuales, seres vivos multicelulares, células, moléculas, áto- 
mos, partículas elementales. Para Oppenheim y Putnam, microrreducir es explicar las propiedades del todo en términos de las propiedades de sus partes (1991: 406-408). Después de todo, un objeto es simplemente la suma de sus partes. Por ejemplo, la química afirma que un pedazo de madera no es otra cosa que el arreglo de varios tipos de moléculas unidos; la física atómica nos dice que las moléculas están unidas por fuerzas; la teoría de partículas afirma que los átomos son conjuntos de partículas elementales, y así sucesivamente (Rohrlich, 1988: 295-296). Oppenheim y Putnam tienen la visión de un estado ideal de la ciencia en que se complete la unidad del lenguaje y la unidad de la explicación (1991: 406). Oppenheim y Putnam aceptaron que su propuesta era una hipótesis de trabajo, basada en argumentos metodológicos y evidencia empírica. Sin embargo, como veremos la propuesta presenta varios obstáculos.

De entrada, la microrreducción enfrenta un problema metodológico: el reduccionismo presupone las explicaciones de mayor nivel. Como vimos arriba, la meta de la microrreducción es el completo reemplazo de las explicaciones de mayor nivel por una de menor nivel. Esto significaría que las explicaciones de menor nivel continuaran a ese mismo nivel inferior. Pero los intentos reduccionistas muestran el uso continuo de términos e información de nivel superior.
Consideremos la teoría neurológica que utiliza la información sobre la "conciencia" y la "memoria". La información y los términos típicamente pertenecen a lo que usualmente nos referimos como estados psicológicos. Como puede inferirse, en la explicación reducida hay una implícita explicación de nivel superior. Una situación análoga sucede con las ciencias sociales, en las que el programa reduccionista requiere que los fenómenos sociales sean expresados con referencia exclusiva a individuos. Términos como "maestro", "preso", "soldado", "ciudadano", etcétera, podrían ser empleados en la reducción individualista. Aparentemente, se refieren a individuos, pero implícitamente se está apelando al nivel sociológico. Estos términos pueden tener significado solo cuando son entendidos en un contexto social, lo que significa una apelación al mayor nivel. Un "ciudadano" desprovisto de las instituciones sociales no es realmente un "ciudadano", como un "maestro" no es un "maestro" sin escuela (Kincaid, 1986: 499). En las microrreducciones biológicas también encontramos que las explicaciones bioquímicas utilizan términos $\mathrm{o}$ funciones que presuponen hechos biológicos.

El caso de la biología nos lleva a analizar una siguiente dificultad para las microrreducciones: el problema de las realizaciones múltiples. Si la bioquímica como un nivel reducido nece- 
sita emplear la función biológica es porque los acontecimientos explicados por la teoría de mayor nivel (biológica) pueden ser realizados (instanciados) por indeterminado número de tipos de entidades de nivel inferior (bioquímico). La reducción del sistema inmunológico a la bioquímica nos proporciona una buena ilustración. El término "anticuerpo" pertenece al vocabulario biológico. Cuando un cuerpo extraño, tal como una bacteria, ingresa en el sistema biológico, el anticuerpo se une con el antígeno - la bacteria- para inducir su remoción. Los anticuerpos existen en los niveles biológicos -células, órganos, organismo- y tienen potencialmente millones de diferentes estructuras químicas. Una caracterización bioquímica del anticuerpo sería ciertamente falsa, porque no es la estructura bioquímica - una proteína- lo que hace de una proteína un "anticuerpo", sino que la proteína tiene una cierta secuencia de aminoácidos que permite que se identifique como un antígeno. Las secuencias tienen una estructura física única y pueden ser encontradas en muchas estructuras de tipos de anticuerpos (Kincaid, 1990: 585).

La conclusión es clara. El tratar de reducir un término como "anticuerpo" no nos conduce a una explicación fructífera. Precisamente lo que caracteriza a la entidad "anticuerpo" es su realizabilidad múltiple. El valor de la irreducibilidad de las explicaciones biológicas es que la biología reúne -a través de la descripción funcional- los fenómenos que se nos aparecen como diversos. Una única explicación bioquímica nos llenaría de detalles pero no nos traería una unificación de los fenómenos. En una vena similar, Garfinkel, argumentando en contra de la reducción, sostiene que las microrreducciones, por su detallismo, en último término, no explican un fenómeno: "La microexplicación incluye datos que son irrelevantes para el resultado y por lo tanto sepulta irreconociblemente a la explicación. Nos proporciona una vergüenza de riquezas y en consecuencia, es menos útil" (1981: 56). El proceso adaptativo de la evolución produce diversas estructuras que comparten una función similar; diferentes mecanismos bioquímicos producen similitudes funcionales. La biología toma esas similitudes para explicar los fenómenos que, de otro modo, tendrían poco en común: allí donde el enfoque bioquímico ve diversidad, la biología revela una importante unidad.

Recientemente se ha sugerido que no se ha advertido acerca de la incompatibilidad entre el microrreduccionismo y la mecánica cuántica (Scharf, 1989). Como se dijo anteriormente, la microrreducción supone que los elementos componentes sean explicados en términos de sus partes. Estos términos deben ser microrreducidos, de tal manera que el vocabulario del elemento compuesto es expresado en el vocabulario de sus partes. Scharf observa 
que un aparato de medición, con sus efectos asociados con la medición cuántica, "no es analizable en términos de partículas elementales de la que está compuesto" (1989: 602). El término “aparato de medición" es una noción primitiva en la teoría cuántica, y por lo tanto, evade una definición aludiendo partículas elementales. Sin embargo, el aparato de medición es un objeto macroscópico y un elemento compuesto de partículas elementales.

Cuando se proporcionan explicaciones en la mecánica cuántica, estas involucran una referencia a la medición. Por ejemplo, de acuerdo con la teoría ortodoxa de la mecánica cuántica, los cambios en el sistema microfísico a través del tiempo ocurren en una de dos formas. Primero, en ausencia de la interacción de la medición, el sistema se comporta de una forma determinista, siguiendo la ecuación de Schrödinger y el estado del sistema en $t_{2}$ puede ser predicho sabiendo el estado del sistema en $\mathrm{t}_{1}$. Segundo, si hay una medición del sistema, entonces el sistema se comporta de forma incontrolable e indeterminista, de tal modo que cualquier predicción de un estado futuro involucra proyecciones indeterministas. Como vemos, la descripción de un cambio en la mecánica cuántica ha empleado un aparato de medición - un objeto macrocóspico con propiedades macroscópicas-que interactúa con el microsistema cuántico (Scharf, 1989: 602-605).
Lo que hace interesante el caso de la mecánica cuántica es que frecuentemente la literatura del reduccionismo se concentra en la posibilidad de reducir las ciencias de mayor nivel hacia las de menor nivel. Pero rara vez se menciona el problema del reduccionismo en la misma ciencia básica. De hecho, esto no es un problema muy discutido en la física. Existe un reconocimiento implícito que la física es la más fundamental de las ciencias y que por lo tanto, está encima de las menos fundamentales. Sin embargo, aquí tenemos un caso en las ciencias físicas que va en contra de las presuposiciones del microrreduccionismo. Nótese cómo Oppenheim y Putnam se basan en ciertas suposiciones:

Creemos que la presuposición de que la ciencia unitaria puede ser obtenida a través de la microrreducción acumulativa se recomienda a sí misma como una "hipótesis de trabajo". Esto es, creemos que es de acuerdo con los estándares razonables del juicio científico que se acepta tentativamente esta hipótesis y para trabajar sobre la presuposición que habrá progreso ulterior en esta dirección, sin aseverar la verdad de lo que se ha establecido, o negando que el éxito finalmente se nos escapará (1991: 408).

$\mathrm{Si}$, como Oppenheim y Putnam sostienen, el programa depende de la evidencia metodológica empírica, parece muy relevante considerar las conclusiones de la física acerca de la mecánica cuántica. El juicio de los físicos acerca de la interpretación de la mecánica cuántica parecería ser "los están- 
dares razonables del juicio científico" a que los dos autores se refieren. Y si este es el caso, se podría sospechar que el programa de la unidad de la ciencia basado en la microrreducción es problemático, a menos, por supuesto, que los microrreduccionistas defiendan la microrreducción universal a pesar de los contraejemplos.

Lo que he querido demostrar es que el programa de microrreducción no solo recibe poco apoyo de las ciencias de nivel superior, sino también de las ciencias físicas. Y recordemos que la microrreducción es considerada el modelo para el programa de unificación de las ciencias. Pero, ¿por qué debiera verse la unidad de la ciencia a través de ese modelo? ¿Por qué para obtener una explicación adecuada debiera expresarse la complejidad de la realidad en un solo vocabulario? Quiero sugerir que la unidad de la ciencia no tiene por qué estar relacionada necesariamente con el programa reduccionista pero con uno de complementariedad a diferentes niveles. Bajo esta perspectiva, la unidad no se ve como una exclusión o reemplazo de niveles superiores de explicación sino como una interrelación entre diferentes niveles.

Consideremos la diferencia entre la física newtoniana y la física relativista. Aun cuando pertenecen al mismo nivel de explicación, puede ayudarnos a ilustrar la visión que estoy defendiendo. Los términos teoréticos son bastante diferentes en cada teoría (v.g., grave- dad). Uno podría optar por descartar las explicaciones newtonianas y explicar totalmente dentro del vocabulario relativista. Pero, como sabemos, los científicos explican una gran cantidad de fenómenos utilizando la física newtoniana y la investigación actual hace uso del modelo newtoniano. La física newtoniana es considerada adecuada para la explicación de fenómenos dentro de unos límites específicos. Las explicaciones relativistas no son consideradas siempre como superiores en comparación con las newtonianas, dentro del rango de esta última. Y, posiblemente, en el futuro, los nuevos modelos proporcionarán explicaciones de fenómenos que no pueden ser abordados por la física relativista.

En la biología tenemos un claro ejemplo de conexión entre los niveles. La biología molecular es resultado de la integración de la bioquímica y la biología, y sin embargo, la biología permanece irreducible. Tal integración demuestra que el no-reduccionismo es fructífero: las explicaciones biológicas sugieren posibles rutas para la investigación en bioquímica, puesto que la bioquímica utiliza la información biológica del nivel celular. Inversamente, el estudio de los procesos moleculares subyacentes a los fenómenos bioquímicos pueden conducirnos a confirmar la superviniencia de los hechos biológicos sobre los bioquímicos, revelando los mecanismos en juego. 
Otra área que ilustra la conexión inter-nivel es la psicología clínica y la psiquiatría (Harris \& Schaffner, 1992). Es relativamente reciente el efecto que la investigación de las bases genéticas en el campo de la enfermedad mental ha tenido sobre la psicología clínica. Los fundamentos biológicos proporcionan una fuente de enriquecimiento para la explicación de la conducta anormal. La elucidación de los mecanismos moleculares en tales enfermedades como la esquizofrenia tendrá un impacto profundo en la manera como se determina la etiología y patogénesis general. Sin embargo, así como el progreso aumenta en la biología de las enfermedades psiquiátricas, existe un reconocimiento generalizado acerca de la importancia del apoyo proveniente de los modelos conductuales, sociales y culturales que son jerárquicamente superiores. Como afirman Harris y Schaffner:

Es improbable que la enfermedad psiquiátrica sea completamente explicada por la genética molecular sin recurrir a los factores ambientales, sociales y culturales. Los modelos de la enfermedad mental que serán útiles tendrán que integrar la información genética a teorías de mayor amplitud siguiendo las líneas de la "incrustación" de las teorías genéticas en las teorías ambientales y socioculturales (1992: 130).

Tomemos la esquizofrenia como un ejemplo. Es una enfermedad que tiene factores biológicos, sociales y ambientales, que sugieren que existe en dife- rentes niveles de organización. Así, una perspectiva de nivel múltiple de la esquizofrenia evita un miope enfoque único de esta compleja enfermedad, que no solo produciría una versión incompleta, sino posiblemente falsa de la enfermedad.

Los casos descritos pueden proporcionarnos un marco de referencia para la unidad que tengo en mente. La diversidad de la realidad, dada la evolución cósmica, requiere una apropiada construcción mental de la organización jerárquica de los fenómenos para aprehender las diferentes propiedades de los objetos (sean átomos, la tierra, las células, los organismos, los grupos sociales, etcétera). Inevitablemente, las diferencias de propiedades requieren un enfoque pluralista en la explicación, no solo de orden epistemológico, sino también ontológico. El proyecto reduccionista presupone una explicación ontológica monista. Pero esta presuposición no toma en cuenta no solo la teoría científica sino la práctica científica. Consideremos nuevamente la diferencia entre la física newtoniana y la relativista. Una habla de espacio curvo, la otra de fuerzas gravitatorias. Preguntar cuál metafísica es la correcta sería simplemente no entender el punto de la explicación física. Aquí hay dos ontologías. Cada una proporciona una intuición en la explicación de los fenómenos. Este ejemplo es análogo de muchos que encontramos en las diferentes ciencias. ¿Por qué debiéramos esperar 
que todo fuera explicado con una misma ontología? La historia reciente de la física nos demuestra la importancia de reconocer una metafísica pluralista. La controversia acerca de la mecánica cuántica nos proporciona una luz para darnos cuenta del mundo en que vivimos. Y el mundo no parece estar de acuerdo con las expectativas de algunos reduccionistas. Como lo señala McMullin:

\footnotetext{
¿Por qué hemos pretendido que la ontología del micromundo sea igual a la del macromundo? La tercera regla de Newton para filosofar (que decretaba que el macromundo debiera ser semejante al micromundo en todos sus detalles esenciales) no ha sido más que una esperanza piadosa (1984: 13).
}

Una metafísica pluralista no es incompatible con la unidad de la ciencia. Al contrario, puesto que no hay una última verdad científica conocida, la integración entre los niveles de las teorías puede demostrar ser una explicación más fructífera y acertada, que de otro modo se nos presentaría un mundo de eventos inconexos.

\section{EL ROL DE LA PSICOLOGÍA POPULAR COMO UN COMPONENTE CAUSAL EN LAS TEORÍAS PSICOLÓGICAS CIENTÍFICAS}

Tanto para los defensores como para los críticos de la psicología popular, las explicaciones de esta no serán reducibles con las explicaciones neurofisiológicas. Los críticos lo consideran como una prueba del fracaso, mientras que los defensores lo ven como una prueba de la autonomía de la psicología popular. Los críticos tienen una visión negativa acerca de la posibilidad de la psicología popular de proporcionar una teoría empíricamente solvente y fructífera. Los defensores toman una posición más optimista.

Explicaré por qué tiene sentido adoptar una posición de defensa del rol la psicología popular en la psicología científica.

Considero que el conocimiento de la psicología popular y la teoría de la psicología popular (una basada en la eficacia causal de las actitudes proposicionales como la creencia y deseo), es usado en dos formas en la psicología. El primer uso se basa en la suposición de la psicología contemporánea que las creencias populares y las teorías populares tienen una sustantiva influencia en los juicios y en el comportamiento. En efecto, el razonamiento popular es, en parte, el objeto de estudio de algunas disciplinas dentro de la psicología, como la psicología social y la cognición social. En este sentido, los modelos psicológicos sociales utilizan invariablemente alguna forma de las estructuras cognitivas típicas de la psicología popular. Pero hay un segundo uso, en el que la psicología popular se convierte en un recurso para ayudar a construir una teoría psicológica que inclusive va más allá de los alcances de la psicología popular. Este segundo uso parte de 
la teoría popular que interpreta que las personas actúan como resultado de un comportamiento intencional y no como una serie de eventos. En otras palabras, el segundo uso toma en cuenta la misma teoría popular acerca de la intencionalidad de la acción humana en la teoría científica.

En el primer uso no se considera importante la validez o el valor de verdad de las proposiciones que constituyen parte de las actitudes proposicionales. Las creencias populares pueden ser patentemente falsas, y sin embargo, tener una influencia causal determinante en el comportamiento subsiguiente. Por ejemplo, la creencia de que los hombres son mejores que las mujeres en habilidad matemática es una falacia, sin embargo, la atribución de esta creencia podría explicar por qué un individuo prefirió contratar a un hombre y no a una mujer para un puesto que requería conocimiento estadístico. Este tipo de uso de la psicología popular, que emplea esquemas cognitivos y creencias particulares, se encuentra en el estudio de la memoria, de los estereotipos, de las impresiones de la personalidad, emoción, autopercepción, persuasión, depresión, roles sexuales y de género, comportamiento grupal, conformidad, prejuicios, agresión y muchos otros más.

En algunos modelos psicológicos se da un peso a la estructura cognitiva, al proceso y al contenido de la cognición popular, que significa tomar en cuenta las creencias particulares o los juicios sociales que un individuo tiene. En efecto, se asume en estudios como los mencionados, que si se presta atención a las creencias y atribuciones podemos tener el conocimiento de los esquemas cognitivos subyacentes. Tómense, por ejemplo, los rasgos atributivos o disposiciones. Los trabajos iniciales que se referían a estos procesos tomaban una perspectiva cognitiva estándar y consideraba a las disposiciones como una clase homogénea. Sin embargo, trabajos posteriores demostraron que los esquemas subyacentes de las relaciones entre los criterios conductuales y la atribución de disposiciones diferirá de acuerdo con la clase de disposición que se atribuya. Algunas características personales (capacidad para hablar, puntualidad, etcétera) parecen estar asociadas con un esquema de frecuencia en la que la intensidad de la atribución está directamente relacionada con la frecuencia del comportamiento. Hay evidencia de que otras características (habilidad y moralidad, por ejemplo) son asimétricas con respecto a criterios conductuales positivos y negativos. Por ejemplo, las personas hacen atribuciones extremas de deshonestidad sobre la base de un ejemplo de comportamiento deshonesto, pero hacen atribuciones muy leves sobre la base de un ejemplo de comportamiento honesto. El uso primero de la psicología popular no pretende ningún compromiso con el valor de verdad ni con la credibilidad cientí- 
fica de las estructuras del conocimiento. Aun cuando las proposiciones creídas resulten ser falsas, los psicólogos usan las teorías causales de la psicología popular para explicar cómo las personas elaboran juicios acerca de las relaciones entre las personas o hacen atribuciones causales. $\mathrm{Y}$ en este sentido, no tiene lugar la analogía que compara la relación de la física popular con la física científica, puesto que la segunda puede prescindir totalmente de las intuiciones de la persona ordinaria, mientras que en la psicología, como he querido mostrar, no se puede, si se quiere entender a los seres humanos.

Es probable que este uso quede restringido al uso actual en la psicología científica, a saber, la psicología social y de la personalidad. Y posiblemente estarán ausentes o serán inexistentes en otras áreas como la neuropsicología, la percepción auditiva o quizás el aprendizaje del lenguaje. Con lo cual, por un lado, afirmamos el dominio de una teoría como la psicología popular, y también se establecen sus límites, puesto que es claro que las teorías de la psicología científica que incorporarán categorías de la psicología popular producirá concepciones e ideas que irán mucho más allá del sentido común, cuando no en contra.

En el segundo uso se asume la veracidad, no del contenido de las actitudes proposicionales, sino de la teoría de la psicología popular, tomándola como válida, o al menos plausible. Lo intere- sante de esto último es que la psicología científica, particularmente la psicología social, necesita prestar atención a la psicología popular, puesto que las teorías populares juegan un rol causal en la producción en los mismos comportamientos y cognición que tanto interesan a los psicólogos sociales. Esto es un punto importante que demarca decisivamente a la psicología de otras ciencias. Por supuesto, la gente ordinaria tiene teorías populares acerca de la física, biología o paleontología, pero tanto los físicos, como los biólogos o los paleontólogos no tienen que prestar atención a tales teorías en el desarrollo de sus teorías científicas, porque estas creencias no tienen influencia causal en los fenómenos en cuestión. Esta diferencia nos permite, precisamente, sostener la importancia del rol de la psicología popular en el desarrollo de las teorías psicológicas que poco se ha reconocido.

Es quizás en el área de la ciencia cognitiva donde vemos el mayor uso de las categorías de la psicología popular, a saber, la postulación de estados mentales que son causalmente relevantes, como, por ejemplo, cuando decimos: "fui a buscar una cerveza en la refrigeradora porque creía que la cerveza está en la refrigeradora y quería tomar una cerveza". La ciencia cognitiva explota este uso causal de las entidades (creencias y deseos) en la explicación de las conductas. Esto supone naturalmente la visión computacional de la mente. En 
efecto, de acuerdo con algunas versiones (Fodor, 1974), las actitudes proposicionales tienen representación y las operaciones causales entre las distintas actitudes proposicionales representadas tienen lugar en un substrato material, por ejemplo, el cerebro. De acuerdo con Fodor, las actitudes proposicionales son funcionalmente discretas, estados semánticamente interpretables que juegan un rol causal en los eventos psicológicos y, en último término, en la conducta observable.

Esta versión computacional ha sido objeto de muchas críticas por parte de los eliminativistas (Churchland, Stich y otros) que consideran que el modelo computacional discreto es incorrecto, puesto que los modelos actuales, que son conexionistas, postulan redes neurales en el que la información se distribuye a través de toda la red y no en forma de la arquitectura cognitiva computacional tradicional que opera siguiendo representaciones discretas y funcionalmente distintas (como lo haría la teoría de la psicología popular).

Pero esta crítica asume que si el conexionismo es correcto, entonces el rol de la psicología popular es incorrecto y falso. Sin embargo, esto habría que probar, puesto que no se sigue necesariamente que las actitudes proposicionales como creencias y deseos estén localizadas en partes específicas o espacios de una red conexionista. Nada impide, a priori, que las actitudes proposicionales estén representadas o estén asociadas con un patrón completo de activación, con todos sus conjuntos de inputs, outputs y mecanismos de medición de activación. Más aún, los modelos conexionistas, tales como patrones de activación, podrían tener poderes causales para iniciar comportamientos o cualquier otra red del sistema. Finalmente, los patrones de activación pueden ser derivados de los inputs, que son semánticos, y conducir outputs, que también son semánticos. En suma, los modelos conexionistas son suficientemente capaces de sostener las características de las operaciones que están asociadas a la psicología popular, y que podrían conservar sus mismas características, a saber, funcionalmente discretas, semánticamente interpretables y tener estatus causal.

Por último, la psicología popular representa un modo de entendernos como humanos, puesto que asume que actuamos por razones que pueden ser equivalentes a causas teleológicas, como cuando decimos que realizamos un acto por algún motivo. Según los eliminativistas debiéramos abandonar las propiedades asociadas a las actitudes proposicionales y, por lo tanto, a todas las explicaciones causales que se le asocian. Esto supondría eliminar a los agentes en este mundo, puesto que todo agente es una persona que actúa por una razón (personas cuyo comportamiento es causalmente explicado en términos de actitudes proposicionales). En este sentido, parecería que el argu- 
mento eliminativista es uno de autorrefutación, puesto que al eliminar las explicaciones que dan sentido a la actuación por las razones, eliminan a las mismas personas eliminativistas. Las actitudes proposicionales no son comparables al flogisto, como sostienen sus detractores, puesto que después del descubrimiento del oxígeno, no encontramos rastro alguno del flogisto, pero debemos preguntarnos, si acaso no es cierto que encontramos los conceptos de actitudes proposicionales ejemplificados en los estados subjetivos íntimamente familiares, y no ha habido un test empírico en el que se haya demostrado que los conceptos de las actitudes proposicionales deje de ser ejemplificado. Y eso porque somos seres subjetivos, que actuamos por razones que la psicología popular parece haber entendido muy bien desde siempre. ¿Por qué la psicología científica tendría que ser tan diferente?

\section{REFERENCIAS}

Blackburn, S. (1991). Losing your mind: physics, identity and folk burglar prevention. En: J. Greenwood (Ed.). The future of folk psychology: intentionality and cognitive science. Cambridge: Cambridge University Press.

Churchland, P. (1980). Plasticity: conceptual and neuronal. Behavioral and Brain Sciences, 3.

Churchland, P. (1981). Eliminative materialism and the propositional attitudes. Journal of Philosophy, 78, 67-90.

Churchland, P. (1988). Matter and consciousness. Cambridge, Mass.: MIT Press.

Churchland, P. S. (1986). Neurophilosophy. Cambridge, Mass.: MIT.

Davidson, D. (1970). Mental events. Reimpreso en (1980). Essays on Actions and Events. Oxford: Clarendon Press.

Feyerabend, P. (1963). Materialism and the mind-body problem. Review of Metaphysics, 17.

Fodor, J. (1968). Psychological Explanation. Nueva York: Random House.

Fodor, J. (1998). Cognitive Science: Where Cognitive Science Went Wrong. Oxford: Clarendon Press.

Fodor, J. (1974). Special Sciences, or The Disunity of Science as a Working Hypothesis. Synthese, 28, 77-115.

Garfinkel, A. (1981). Forms of Explanation: Rethinking The Questions in Social Theory. New Haven: Yale.

Harris, H. \& Schaffner, K. (1992). Molecular Genetics, Reductionism, and Disease Concepts in Psychiatry. The 
Journal of Medicine and Philosophy, 17, 127-153.

Kincaid, H. (1986). Reduction, Explanation and Individualism. Philosophy of Science, 53, 492-513.

Margolis, J. (1987). Science without unity: Reconciling the Human and Natural Sciences. Oxford: Basil Blackwell.

McCloskey, M. (1983). Naive theories of motion. En D. Gentener \& A. L. Stevens (Eds.), Mental Models (pp. 299324). Hillsdale, N. J.: Lawrence Erlbaum.

McMullin, E. (1984). A Case for Scientific Realism. En: J. Leplin (Ed.). Scientific Realism. Berkeley: University of California Press.

Neisser, U. (1967). Cognitive psychology. Englewood Cliffs, N. J.: Prentice-Hall.

Oppenheim, P. \& Putnam, H. (1991). Unity of Science as a Working Hypothesis. En: R. Boyd, P. Gasper \& J. D. Trout. The Philosophy of Science. Cambridge, MA: MIT, 403-427.

Popper, K. (1974). Scientific reduction and the Essential incompleteness of all science. En: F. Ayala and T. Dobzhansky (Eds.). Studies in the Philosophy of biology. Londres: Macmillan.
Ravenscroft, I. (2004). Folk psychology as a theory. En: Stanford Encyclopedia of Philosophy. <http://www.plato.stanford.edu/ entries/folkpsych-theory/>.

Rohrlich, F. (1988). Pluralistic Ontology and Theory Reduction in the Physical Sciences. British Journal for the Philosophy of Science, 39, 295-312.

Rorty, R. (1970). In defense of eliminative materialism. Review of Metaphysics, 24.

Ryle, G. (1949). The Concept of Mind. Chicago: The University of Chicago Press.

Sarkar, S. (1992). Models of Reduction and Categories of Reductionism. Synthese. 91, 167-194.

Scharf, D. (1989). Quantum Measurement and the Program for the Unity of Science. Philosophy of Science, 6, 601623.

Sellars, W. (1968). Science and metaphysics. Londres: Routledge.

Stich, S. (1985). From Folk Psychology to Cognitive Science: A Case against Belief. Cambridge, Mass: MIT Press.

Von Eckardt, B. (1994). Folk Psychology. En: S. Guttenplan (Ed.). A Companion to the Philosophy of Mind (pp. 300307). Oxford: Blackwell. 